AKTUELLE PROJEKTE ZUR OEKOSYSTEMFORSCHUNG IM SCHWEIZERISCHEN NATIONALPARK

\section{Einleitende Bemerkungen}

Seien es Leute wissenschaftlicher Gremien oder touristische Besucher des Nationalparks, sie betrachten dieses $\mathrm{Na}-$ turreservat vorwiegend als Raum einheimischer Tiere. In zweiter Linie erkennt man allenfalls den wert oder den Reiz der naturnahen Vegetation, aber uber die grossen Besonderheiten der geomorphologischen Landschaftsgestaltung ist man sich kaum je bewusst. Gerade sie sind es aber, welche den Rahmen dieses Oekosystems primär abstecken. Die folgenden Ausführungen zielen also darauf hin, die darin wirksamen Prozesse und vielschichtigen Zusammenhänge aus physisch-geographischer sicht aufzudecken und das heutige Landschaftsgefüge modellhaft nachzuzeichnen. Dabei stellen sich drei Teilfragen:

1. Wie ist methodisch und organisatorisch vorzugehen ?

2. In welchen Bereichen, räumlich und thematisch gesehen, liegen die Wesenszüge der seit 1914 streng geschützten Landschaft begründet ?

3. Wie intensiv verlaufen einzelne Prozesse und Veränderungen heute ?

\section{Methodik, Begriffe, Organisation}

Methodisch gilt es, den $168 \mathrm{~km}^{2}$ grossen Nationalpark einerseits als ganzheitliches System $z u$ erfassen, andererseits in sinnvolle Teilregionen $\mathrm{zu}$ gliedern. Da seit fast 80 Jahren die land- und forstwirtschaftliche Nutzung entfällt, ist eine besondere Einheitlichkeit bereits vorgegeben. Doch nicht nur diesbezüglich stellt der Nationalpark eine regionale Einheit dar, sondern auch hinsichtlich seiner Geologie, ist er doch von Dolomiten der Ostalpinen Decken beherrscht (Fig. 1). Ebenfalls klimatisch nimmt der Ofenpassraum eine Sonderstellung ein, denn hier herrscht ein betont trockenes, kontinental gefärbtes Klima mit einer $00^{\circ} \mathrm{C}-J a h r e s m i t t e l$ temperatur auf ca. $2000 \mathrm{~m}$ ü.M. Dies wiederum erzeugt vegetationsgeographische Einschränkungen, kommen doch weitgehend kalkliebende Gebirgspflanzen vor und bis über $2300 \mathrm{~m}$ hoch steigende Arvenund Lärchenbestände (Foto 1). Die hydrologischen Verhältnisse sind in den unterhalb $2000 \mathrm{~m}$ gelegenen subalpinen Bereichen des Nationalparks gestört, da alle grösseren Täler zwecks Energienutzung via Livigno/Spöl nur noch Rest- wasser führen. Dies betrifft aber nicht die ausgedehnte alpine stufe, wo z.B. ausgesprochen viele Quellen anzutreffen sind. Insgesamt kann der Nationalpark von seinen oekologischen Voraussetzungen und seiner Landschaftsdynamik her als weitgehend geschlossene Einheit bezeichnet werden, er stellt somit ein Oekosystem dar. Darunter sind naturnahe und allenfalls anthropogen umgestaltete Landschaften $z u$ verstehen, die mehr oder weniger in sich geschlossene, aber beliebig grosse Raumeinheiten darstellen, und worin sich vielfältige biologische und geographische Faktoren gegenseitig ausbalancieren und $\mathrm{zu}$ einem Gleichgewicht einpendeln. Diesem Begriff, dem im Sinne von LESER (1984, S.355) eine funktionale Betrachtung zugrundeliegt, steht ein entsprechender räumlicher Begriff gegenüber: Oekotop. Seine Dimensionen können wie erwähnt je nach Situation festgelegt werden, in praktischen Forschungen wird es sich aber am ehesten um eine Talschaft oder ein grösseres Einzugsgebiet eines Flusses handeln. Allenfalls zerfällt es in einzelne Subsysteme, z.B. Talflanken.

Für die konkrete Forschung im Nationalpark hat es sich als sinnvoll erwiesen, das Oekosystem Nationalpark sowohl räumlich als auch thematisch auf verschiedene Personen aufzuteilen. Gebiete von je ca. $30 \mathrm{~km}^{2}$ werden gezielt auf ihre spezifischen landschaftsformenden

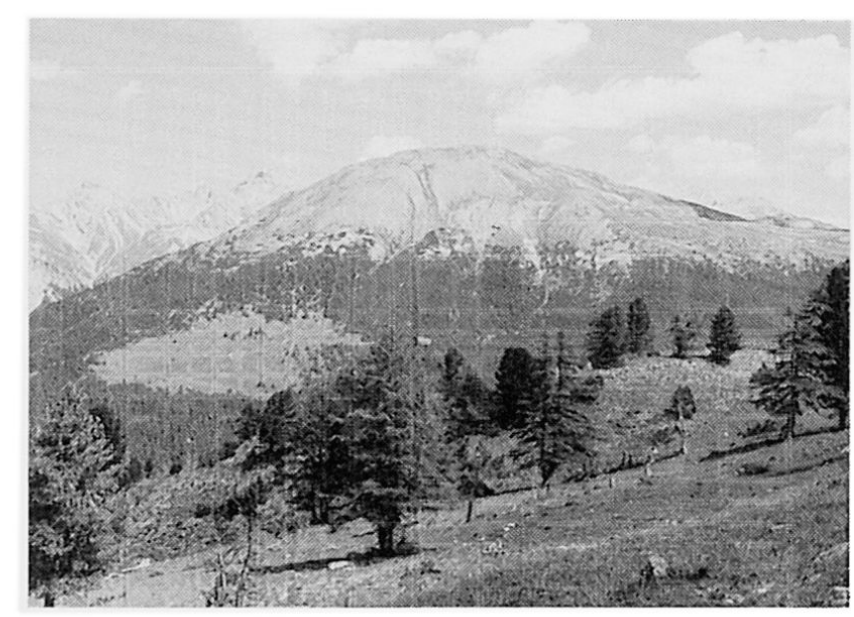

Foto 1: Der Munt La Schera mit Alp La Schera im Bildmittelgrund, gesehen aus dem Val da I'ACqua von SW. Der Föhrenbestand im Vordergrund mit einzelnen Lärchen und Arven befindet sich auf $2150 \mathrm{~m}$ ü.M.

Kurt Graf, Prof., Dr., Geographisches Institut der Universität Zürich-Irchel, 8057 zürich 


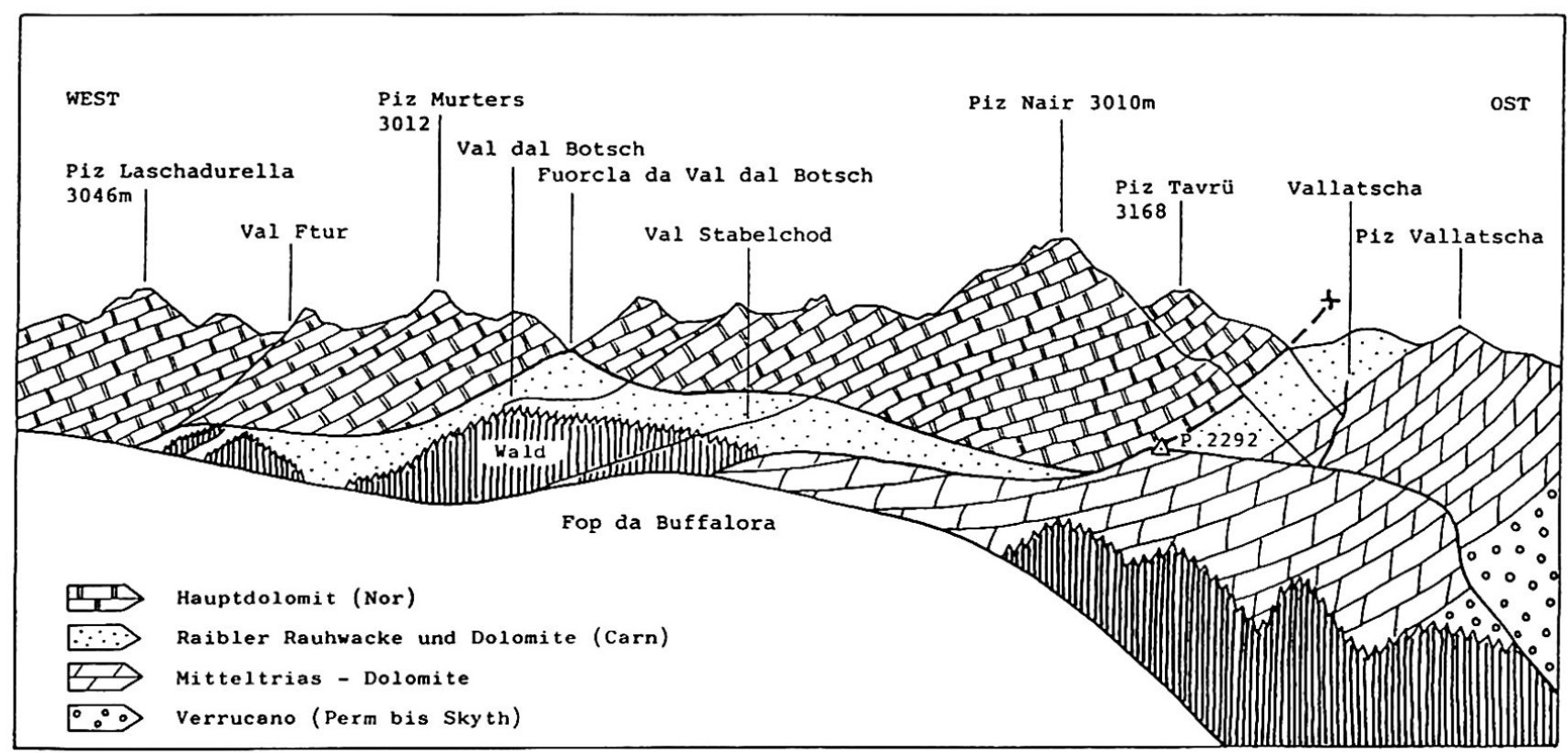

Fig. 1: Die nördliche Flanke des Val dal Fuorn mit ihren geologischen Haupteinheiten, gesehen von der alten Eisenmine Buffalora.

Prozesse und deren Auswirkungen hin untersucht. Diese stehen oder standen in Bearbeitung bei mehreren Leuten am Geographischen Institut der Universität Zürich:

Flückiger Sibylle (in Vorb.), Biomasse, Val dal Spöl

Jäger Peter (in Vorb.), EDV-Konzept, Il Fuorn-Südflanke.

Menz Marius (in Vorb.), Klima, Il Fuorn Reithebuch Jakob (1990), Denudation, Val Tantermozza

Reusser Stefan (1990), Moränen u.a., Il Fuorn-Nordflanke

Stetter Gideon (in Vorb.), Murgänge, Val Cluozza

Ebenso kommen diverse Arbeitsmethoden zum Einsatz:

-Geomorphologische Kartierung im Gelände (in Anlehnung an die GMK-25 nach LESER, aber nur auszugsweise und primär im Massstab 1:10'000)

-Vergleich mit Infrarot-Luftbildern 1:9000 des Sanasilva-Projekts am BUWAL, Flug der Eidg. Vermessungsdirektion vom 7.8.1988

-Auswertung von Protokollblättern der Nationalparkwächter zu Schadenereignissen

-Labormässige Auswertung von Bodenproben in einem Höhenprofil

-Labormässige und mikroskopische Auswertung von Pollenproben

-Vegetationsschadenkartierung und
-Bodenbewegungsmessungen (GRAF 1991, S. 51)

Bei der Auswertung wird es entscheidend sein, die empirisch ermittelten Informationen $z u$ verdichten und $z u$ generalisieren. Mehrere solche Verarbeitungen von Daten stehen zur Auswahl:

- Regionalisierung: Abgrenzen und räumliches Ordnen von Landschaftseinheiten nach bestimmten Kriterien.

- Visualisierung: Zeichnerisches Erfassen von Begriffen, wobei wesentliche Fakten einigermassen naturgetreu symbolisiert sind. Dabei können statische Gegebenheiten (z.B. eine geologische Raumgliederung) oder dynamische Mechanismen ( $z$.B. das Netz von Karstwasserwegen) dargestellt werden.

Als weitere Möglichkeiten graphischer Darstellungen seien genannt:

- Kausalprofil: Skizzierung eines Geländeausschnittes im Aufriss unter Beschriftung einzelner Abschnitte nach verschiedenen Gesichtspunkten.

- Wirkungsschema: Darstellung kausaler Zusammenhänge mit (oft durch Pfeile angedeuteten) verzweigten oder vernetzten Abhängigkeiten. Damit wird ein System veranschaulicht, das aus mehreren Teilen (=Kompartimenten) besteht. Beispiel: Auswirkungen von Starkregen. 


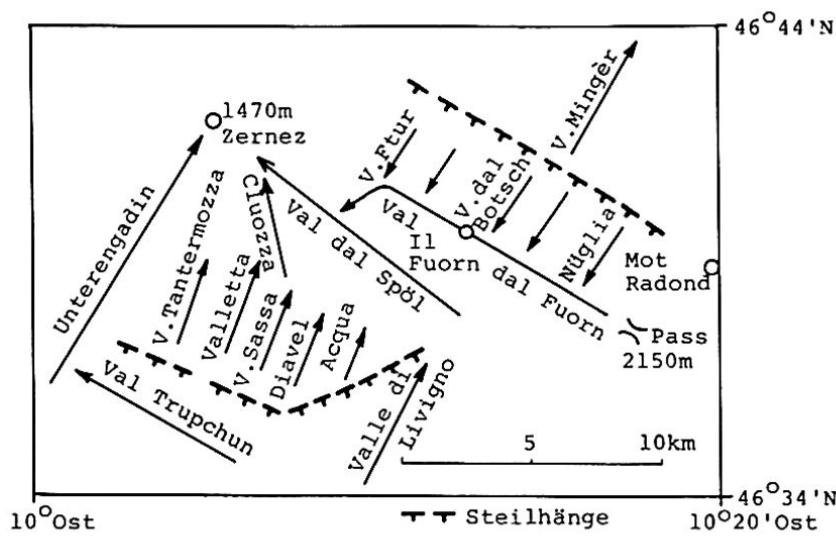

Fig. 2: Das nach Geländekammern gebündelte Hauptentwässerungsnetz im Nationalpark, mit den wichtigsten im Text erwähnten Lokalitäten.
$1800 \mathrm{~m}$. Offensichtlich sind die oberen Talabschlüsse mit 2800-2900 $\mathrm{m}$ zu hoch, als dass die Lokalgletscher sich dort hinüber hätten abdrängen lassen. Vielmehr fülte ihr Eis die Täler und versperrte dem Inngletscher den Zugang. Somit ist die grossräumige Talanlage bis heute in wesentlichen Teilen landschaftsbestimmend geblieben, indem sie die Art des Lockermaterials und damit die aktuellen Abtragungsvorgänge determiniert. Da sich Quellen und vernässte Stellen in typischerweise mit Verrucano bestückten Moränendecken häufen, haben die eiszeitlichen Gegebenheiten also auch ihre hydrologische Bedeutung bis heute behalten.

Im weiteren wird es nun darum gehen, die so ausgelösten Prozesse und Veränderungen im Detail zu beleuchten.

\section{Ergebnisse der Prozessforschung}

Im Zuge der Kartierungen hat sich gezeigt, dass denudative Prozesse bei weitem überwiegen. Man kann sie unterteilen in gravitative Vorgänge (wo sie sich an Felswänden und anderen Steilpartien manifestieren) und denudative i.e.s. (wo flächenhafte Schuttverlagerungen durch ein Medium wie Wasser oder Wind stattfinden). Mit Fig. 3 wird dieser Formenkreis der Denudation i.w.S. veranschaulicht, wie er sich nach einem mehrstündigen Starkregen im Juli 1991 gezeigt hat. Insbesondere soll darin zum Ausdruck gebracht werden, dass mehrere verwandte oberflächenformen stets räumlich und kausal miteinander verknüpft auftreten und somit als Gruppe erfasst werden müssen.

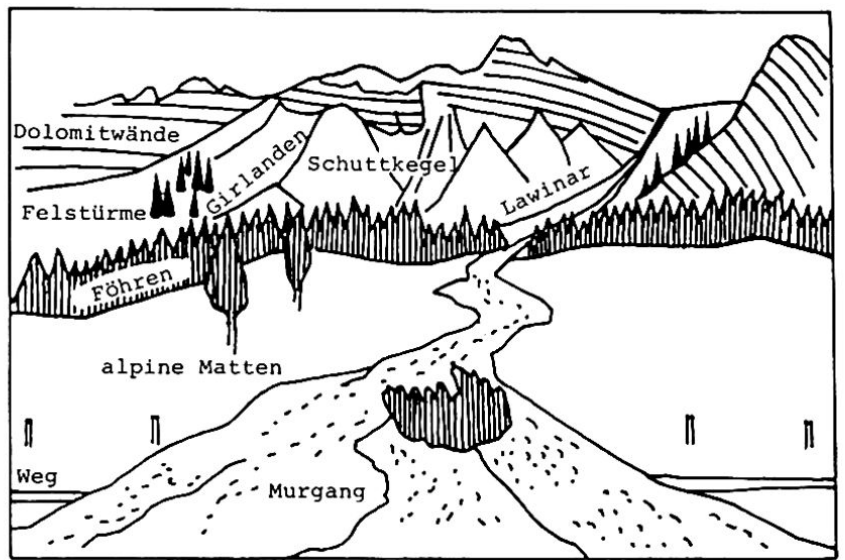

Fig. 3: Ein Beispiel zum denudativen Formenschatz, das sich beim Rastplatz im val dal Botsch von $2000 \mathrm{~m}$ gegen $\mathrm{NW}$ eindrücklich präsentiert. Im Juli 1991 sind der Weg und einzelne Markierungspfosten etwa $1 \mathrm{~m}$ hoch von einer Mure verschüttet worden. 
Zur Denudation i.e.S. zählt die Solifluktion. Dieses frostbedingte Bodenfliessen von durchtränkter Erde wirkt im Nationalpark weitflächig und bringt hier als typische oberflächenformen vor allem Erdströme, Girlanden, Erdkuchen und öfters Bülten hervor. Im Höhenbereich ihres häufigsten Auftretens, zwischen 2000 und $2500 \mathrm{~m}$, wurden oberflächennahe Humusproben einer Pollenanalyse unterzogen und daraus schlüsse auf die standörtlichen Bedingungen gezogen (Fig.4). Generell zeigen die Palynomorphen ein Spektrum mit ausgesprochen viel Baumpollen einerseits, aber auch mit (aus der Pollensumme von 100\% ausgeklammerten) vielen Sporen. Baumpollen ist durch Fernflug eingeweht worden, widerspiegelt also ein schütteres Aufkommen von Lokalvegetation. Hingegen stammen Sporen vorwiegend von örtlich wachsenden Pflanzen wie Botrychium Lunaria (Mondraute) oder Selaginella (Moosfarn); letztere sind zudem noch besonders verwitterungsresistent und lassen damit Folgerungen auf die Ablagerungsbedingungen ziehen. Im speziellen heisst dies für die vier untersuchten Solifluktionsformtypen, dass Bülten (mit ihren akzentuierten Anteilen von Compositen, Gramineen und Botrychium) relativ wenig intensive Verwitterung und Frostbewegung erfahren, hingegen Erdströme und Erdkuchen (gemäss der grossen Zahl Selaginellasporen) einem extrem starken mechanischen Stress ausgesetzt sind. Ihre Bodenbewegungen finden also intensiv statt, stark wird dementsprechend die Vegetationsdecke geschädigt und aufgerissen, und ihre Lokalitäten (Sättel) sind auch dem Wind und Sonnenlicht besonders ausgesetzt. Girlanden nehmen eine Zwischenstellung ein, was sich real in der grossen ökologischen Bandbreite ihres Auftretens im Gelände äussert.

Betreffend Karst liegen keine Messungen vor, sondern lediglich Feldiveobachtungen. Es ist indessen unschwer herauslesbar, dass Karbonat- und Gipslösungsprozessen im Nationalpark ein grosser Stellenwert eingeräumt werden muss. Zahlreiche Dolinen finden sich etwa an der Südflanke des Munt La Schera $146^{\circ}$ 39 'N/100ll'E, Militärkoordinaten 812 . 200/169.300), ebenso im Val Ftur (REUSSER, 1990, S. 6l; wobei er dies als Kryokarst auffasst). Karren mustern vielerorts Dolomitfels und -blöcke; ihr Auftreten beschränkt sich also keineswegs nur auf reine Kalke. Karstisch mutet auch der obere Abschnitt im Val Tantermozza an, wo der Bach (teilweise wohl durch übermässigen schuttanfall von beiden Talflanken) oberflächlich verschwindet und $\mathrm{l} \mathrm{km}$ weiter südlich rieder als Quelle austritt (REITHEBUCH, 1990, S. 39). Man stellt also häufig ein räumliches Nebeneinander von denudativen und karstischen Prozessen fest.

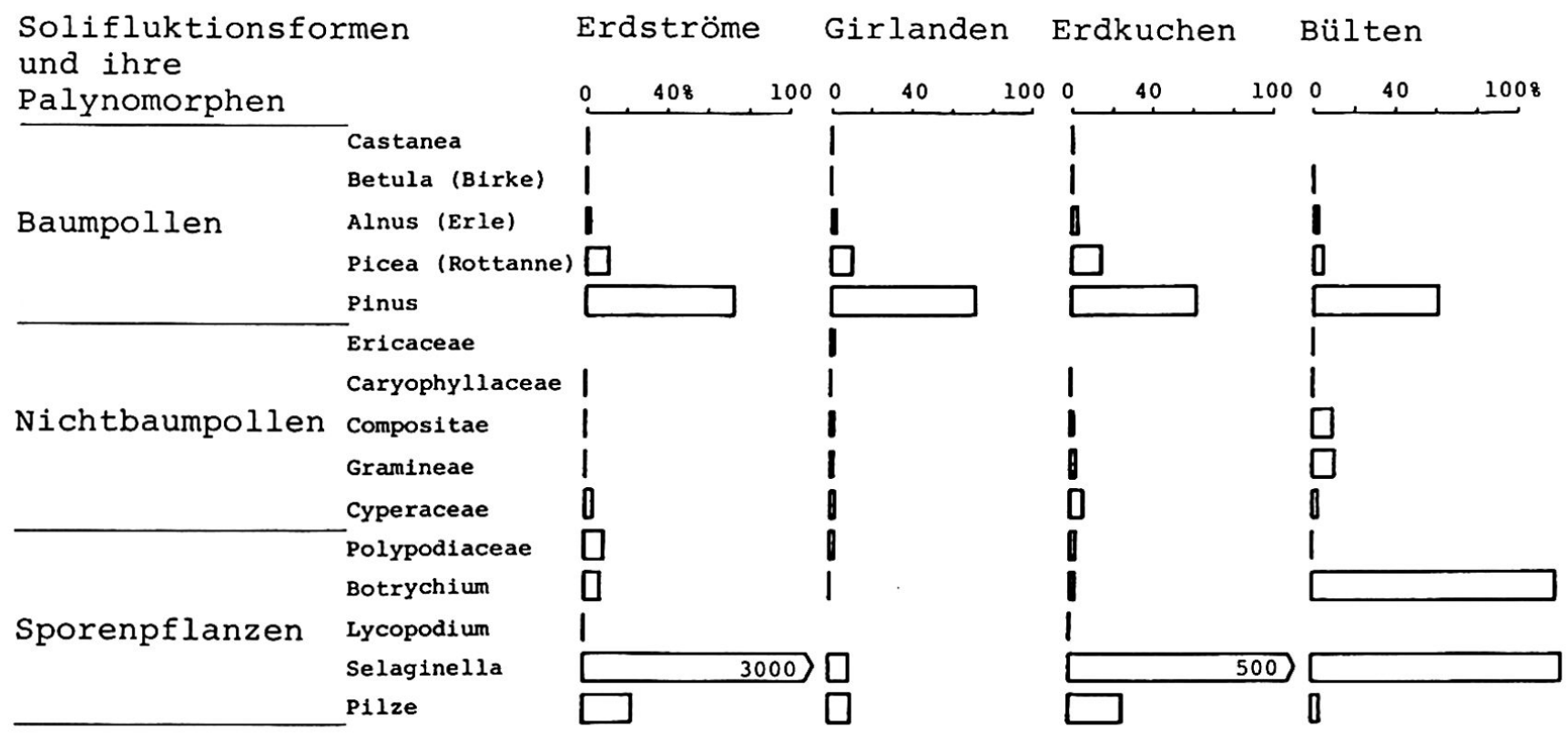

Fig. 4: pollenanalytische Auswertung von 23 oberflächenproben aus verschiedenen Solifluktionsformen. Die gemittelten Werte sind in \% der Pollensumme (Baumpollen und Nichtbaumpollen) angegeben und belegen eine Dominanz von Pinuspollen (Föhren und Arven) sowie von Sporen der Lichtzeigerpflanze Selaginella. 


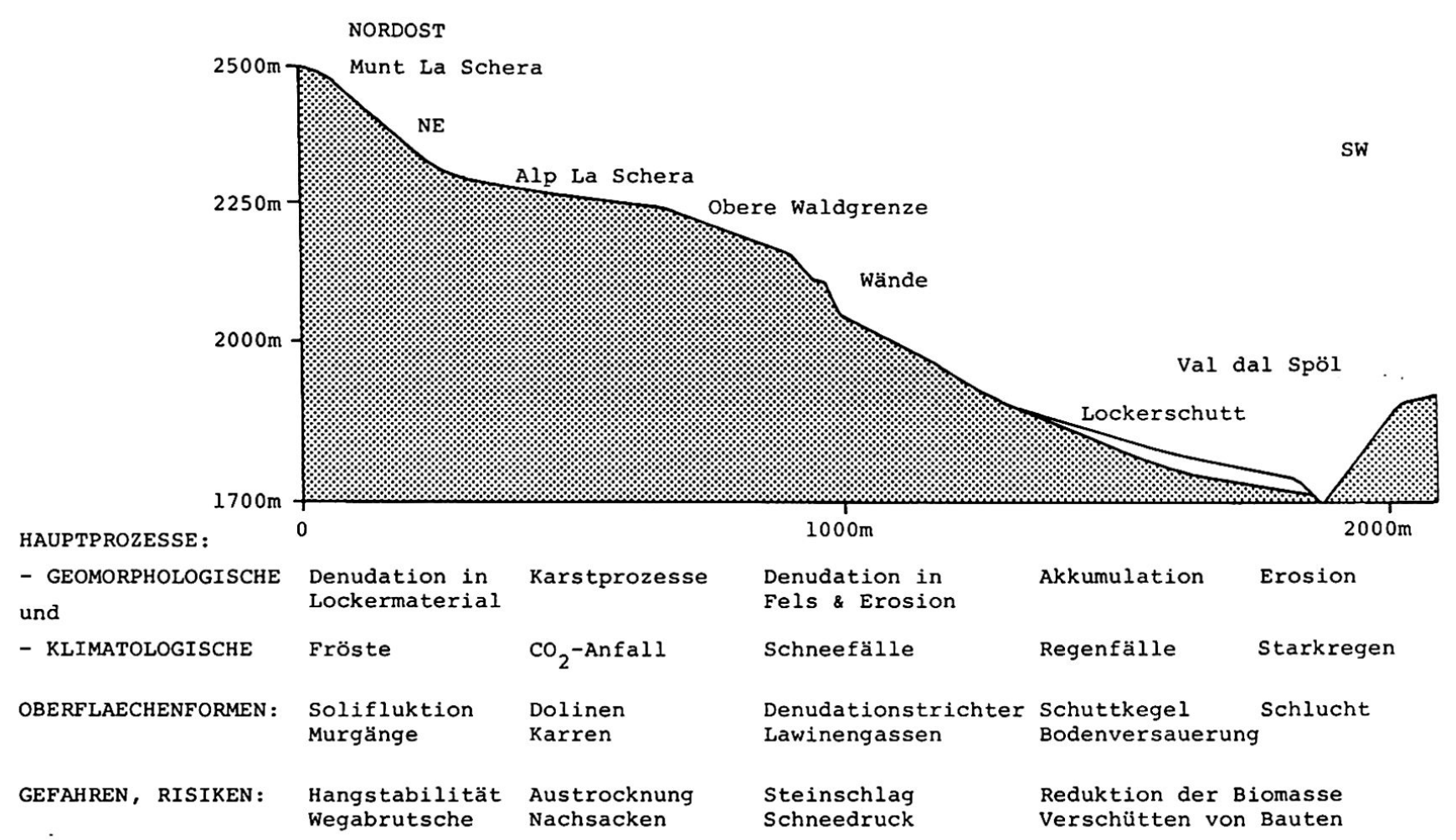

Fig. 5: Dieses Kausalprofil vom Munt La Schera zum Val dal Spöl ist in Foto 1 veranschaulicht und gibt einen Ueberblick zur Landschaftsformung im Nationalpark. Es kann als Schema dieses Oekosystems aufgefasst werden.

Zur Biomasse liegen noch wenige Messdaten vor, und die Aussagen halten sich dementsprechend im qualitativen Rahmen. Im Bereich der subalpinen wälder und alpinen Matten weisen die Böden geringe Feuchtigkeit und Porenvolumen auf, die Werte der Insolation auf den Boden sind hingegen meistens relativ hoch. Somit finden sich darin nur eingeschränkte Populationen Wirbelloser (J.ZETTEL, mal. Nitt.), und auch der Pflanzenwuchs (inkl. Baumzuwachs) ist eher gehemmt. Gesamthaft ist die jährliche biologische Produktion, die Biomasse, also verhältnismässig klein.

In verschiedenen Aspekten hat sich somit bei Untersuchungen im Nationalpark ergeben, dass ganz spezielle Verhältnisse vorliegen. Entsprechend laufen sehr charakteristische Prozesse ab, die nicht nur Bedeutung für das Reservat erlangen, sondern auch direkt ins Umfeld menschlichen Handelns eingreifen. Dass sie oft auch Gefahrenmomente darstellen, sei mit dem zusammenfassenden Kausalprofil von Fig. 5 veranschaulicht. Darin wird am Beispiel des Munt la Schera verdeutlicht, wie sich je nach Gelände verschiedene Hauptprozesse geomorphologisch und klimagesteuert manifestieren.

\section{Literatur}

BOESCH, H. (1969): Berge und Täler der Schweizer Alpen. Verlag Kümmerly \& Frey, Bern: 97136.

DOSSSEGER, R. et al. (1987): Geologische Karte des Schweizerischen Nationalparks 1:50'000. Geol. Spezialkarte Nr. 122.

FURRER, H. (1986): Falten, Felsen und Fliesszungen. In: Schweizerischer Nationalpark, NaturMagazin draussen 42, Harksheider Verlagsgesellschaft, Norderstedt: 36-45.

GRAF, K. (1991): Oekologische Studien auf alpinen Matten in der weiteren Umgebung des Schweizerischen Nationalparks. In: Elsasser, $H$. \& Boesch, M. (ed.): Beiträge zur Geographie Graubündens, Zürich: 50-58.

HANTKE, R. (1983): Eiszeitalter, Band 3. Die jüngste Erdgeschichte der Schweiz und ihrer Nachbargebiete. Ott Verlag Thun: 1-730.

LESER, H. (1984): Zum Oekologie-, Oekosystemund Oekotopbegriff. Natur und Landschaft, 59/ 9: 351-357.

REITHEBUCH, J. (1990): Geomorphologische Kartierung im westlichen Teil des Schweizerischen Nationalparks. Diplomarbeit Univ. Zürich:1-90.

REUSSER, S. (1990): Geomorphologische Kartierung im Massstab 1:25'000 im Schweizerischen Nationalpark. Diplomarbeit Univ. Zürich:1-107. 